\title{
Competing endogenous RNA network analysis reveals potential long non-coding RNAs as predictive biomarkers of gastric cancer
}

\author{
SANFEI PENG ${ }^{*}$, XIANGYANG YIN*, YIZHENG ZHANG, WUNAN MI, \\ TONG LI, YANG YU, JIANWU JIANG, QI LIU and YANG FU
}

Department of General Surgery, The First Affiliated Hospital of Zhengzhou University, Zhengzhou, Henan 450052, P.R. China

Received April 21, 2019; Accepted December 12, 2019

DOI: $10.3892 / \mathrm{ol} .2020 .11351$

\begin{abstract}
Gastric cancer (GC) is one of the most frequently occurring life-threatening malignancies worldwide. Due to its high mortality rate, the discovery of putative biomarkers that may be sensitive and specific to GC is of seminal importance. Long non-coding RNAs (lncRNAs) are non-translatable RNAs whose transcript length exceeds 200 base pairs. The dysregulation of IncRNA expression plays a key role in tumorigenesis and development. In the present study, the expression profiles of lncRNAs, microRNAs and mRNAs of 361 GC tissues (and 32 normal gastric tissues) were downloaded from The Cancer Genome Atlas database. Furthermore, differentially expressed RNAs were analyzed by the DEseq package. Gene Ontology and Kyoto Encyclopedia of Genes and Genomes analyses confirmed some significant dysregulated signaling pathways and target RNAs. As a result, an lncRNA-associated competing endogenous RNA (ceRNA) network was constructed. Kaplan-Meier analysis of the differentially expressed RNAs associated with GC pathogenesis confirmed that the IncRNAs PVT1, HAND2-AS1 and ZNF667-AS1 were potentially associated with the prognosis of GC $(\mathrm{P}<0.05)$. The present study suggests the mechanism of ceRNA networks in GC, and further demonstrates that aberrant lncRNA expression may be used as an effective diagnostic tool (or target) for the prognosis of GC.
\end{abstract}

\section{Introduction}

Gastric cancer (GC) is the fourth most commonly occurring cancer in the world, and the second cause of cancer-associated deaths worldwide. Approximately 990,000 new cases of GC occur every year, with $\sim 738,000$ associated deaths

Correspondence to: Professor Yang Fu, Department of General Surgery, The First Affiliated Hospital of Zhengzhou University, 1E Jianshe Road, Zhengzhou, Henan 450052, P.R. China

E-mail: fuyang@zzu.edu.cn

*Contributed equally

Key words: gastric cancer, competing endogenous RNA, PVT1, HAND2-AS1, ZNF667-AS1 worldwide (1). The high mortality rate of GC is mainly due to limited diagnosis at the earlier phases of the disease, which consequently means that gastric carcinoma is detected when is established at a more advanced stage (2). Hence, the identification of GC-specific tumor biomarkers is warranted for the diagnosis and therapy of patients with GC.

A non-coding RNA (ncRNA) is a type of RNA that is not translated into a protein (3). Due to its structural characteristics, an ncRNA can regulate the expression of an mRNA to promote or inhibit the production of its encoded protein and indirectly exert its biological effects. The ncRNA family includes long ncRNAs (lncRNAs), microRNAs (miR/miRNAs), small interfering RNAs, small nucleolar RNAs and ribosomal RNAs (4). IncRNAs correspond to a class of ncRNAs, which lack open reading frames and are typically longer than 200 base pairs (5). A number of studies have demonstrated that lncRNAs are involved in the pathogenesis of many malignancies and, therefore, could be used as putative biomarkers for the prognosis of these various conditions. The function of IncRNAs can be either cancer-promoting or tumor-suppressing, depending on the level of regulation towards protein-coding genes and/or the chromosome structure $(6,7)$. lncRNAs play roles in various cellular processes, mainly regulating cell growth, differentiation and apoptosis $(8,9)$. miRNA is another type of RNA, 22 nucleotides in length, that can also control the expression of protein-coding genes (10). This type of RNA can modulate gene expression at the post-transcriptional level, by attaching to miRNA response elements along the target transcript $(11,12)$. In 2011, the competing endogenous RNA (ceRNA) theory emerged, introducing novel regulatory mechanisms driven by distinct non-coding RNAs (12). The main idea behind this theory involves the competitive cross-regulation of lncRNAs, mRNAs and miRNAs (13).

Recent studies have revealed that several lncRNAs are associated with the occurrence, metastasis, prognosis and drug resistance of GC (14-16). Notwithstanding this, the roles of IncRNA and miRNA in early and advanced GC are not yet fully understood. Therefore, further studies on the roles of lncRNA in GC could be conducive to the diagnosis and therapy of patients with GC.

In conclusion, the purpose of the present study was to identify novel and specific lncRNAs associated with GC, and to provide insight into the features of IncRNAs as biomarkers for the diagnosis and therapy of patients with GC, thus further improving the prognosis. 


\section{Materials and methods}

Data acquisition from The Cancer Genome Atlas (TCGA). The expression data of mRNA, IncRNA and miRNA in patients with GC were downloaded from TCGA database (https://cancergenome.nih.gov). Specifically, the expression profiles of 393 patients with GC were retrieved from the TCGA-Stomach Adenocarcinoma (STAD) dataset (17) via the University of California Santa Cruz Xena database (https://xenabrowser.net/datapages/). The inclusion criteria were as follows: i) Tissues samples with completed data for analysis and ii) histologic diagnosis is STAD without other malignancies. The exclusion criteria were set as follows: i) Tissues samples without completed data for analysis; ii) histologic diagnosis is not STAD; iii) patients with additional malignancies other than GC and iv) patients who received preoperative chemoradiation. For GC samples containing detailed RNA expression data, the corresponding clinical details were also accessed, including survival time, survival status, sex, staging and other clinical features. A total of 361 cases of GC ( 233 males and 128 females; mean age, 65.66 years; age range, $30-90$ years) and 32 cases of normal (22 males and 10 females; mean age, 68.78 years; age range, 50-88 years) gastric tissue were included in the present study. The detailed clinical features of all patients with $\mathrm{GC}$ are summarized in Table I.

In silico RNA screening. The differentially expressed mRNAs, lncRNAs and miRNAs (DEmRNAs, DElncRNAs and DEmiRNAs, respectively), in both GC and non-tumor tissues, were independently selected using the edgeR package (R language; version 3.28.0; http://bioconductor. org/packages/2.4/bioc/html/edgeR.html) with a threshold of $\mid \log _{2}$ fold-change $\left(\log _{2} \mathrm{FC}\right) \mid>2$ and false discovery rate $($ FDR $)<0.01$ (18). Hierarchical clustering analysis was completed using the pheatmap package in $\mathrm{R}$ (version 1.0.8), in order to estimate the divergence among the differentially expressed RNAs (19).

Differential mRNA enrichment analysis. DEmRNAs were added into the Database for Annotation, Visualization and Integrated Discovery (https://david.ncifcrf.gov/), which takes advantage of GO (http://geneontology.org/) to determine the gene function described in the respective gene profiles. The Kyoto Encyclopedia of Genes and Genomes (KEGG; https://www.kegg.jp/) was also utilized as a platform to investigate the likely functions of the aforementioned genes and their pathways.

Construction of lncRNAs and mRNA co-expression networks. IncRNA-mRNA networks were designed as the basis for IncRNA-mediated control of mRNA abundance. For this, DElncRNAs and DEmRNAs were selected by comparing GC with normal control tissues. To determine the respective RNA contents, a standard selection criteria was set at $\mathrm{P}<0.05$ and fold change $>2$. The lncRNA and mRNA co-expression networks were constructed by correlating DElncRNAs and their respective target mRNAs.

Construction of ceRNA network. TargetScan (http://www. targetscan.org/vert_72/), miRTarBase (http://mirtarbase.mbc.
Table I. Clinicopathological characteristics of patients with gastric cancer.

\begin{tabular}{|c|c|c|}
\hline \multirow[b]{2}{*}{ Characteristic } & \multicolumn{2}{|c|}{ Patients $(\mathrm{n}=361)$} \\
\hline & $\mathrm{n}$ & $\%$ \\
\hline \multicolumn{3}{|c|}{ Age category, years } \\
\hline$\leq 65$ & 161 & 44.6 \\
\hline$>65$ & 200 & 55.4 \\
\hline \multicolumn{3}{|l|}{ Sex } \\
\hline Male & 233 & 64.5 \\
\hline Female & 128 & 35.5 \\
\hline \multicolumn{3}{|c|}{ Pathological stage } \\
\hline I & 63 & 17.5 \\
\hline II & 112 & 31.0 \\
\hline III & 152 & 42.1 \\
\hline IV & 34 & 9.4 \\
\hline \multicolumn{3}{|c|}{ Pathological T stage } \\
\hline Tis & 8 & 2.2 \\
\hline $\mathrm{T} 1$ & 18 & 5.0 \\
\hline $\mathrm{T} 2$ & 75 & 20.8 \\
\hline T3 & 164 & 45.4 \\
\hline $\mathrm{T} 4$ & 96 & 26.6 \\
\hline \multicolumn{3}{|c|}{ Pathological N stage } \\
\hline No & 123 & 34.1 \\
\hline N1 & 96 & 26.6 \\
\hline $\mathrm{N} 2$ & 66 & 18.3 \\
\hline N3 & 76 & 21.1 \\
\hline \multicolumn{3}{|c|}{ Pathological M stage } \\
\hline M0 & 322 & 89.2 \\
\hline M1 & 23 & 6.4 \\
\hline Mx & 16 & 4.4 \\
\hline \multicolumn{3}{|l|}{ Survival status } \\
\hline Alive & 219 & 60.7 \\
\hline Dead & 142 & 39.3 \\
\hline
\end{tabular}

Tis, carcinoma in situ; Mx, distant metastasis is unknown.

nctu.edu.tw/php/index.php) and miRDB (http://mirdb.org/) databases were used to predict target mRNAs (combined with DEmiRNAs). The prediction analysis of lncRNA binding to DEmiRNAs was performed using miRcode software (version 11; http://www.mircode.org). Datasets were intersected in order to strengthen the correlations between mRNAs and DEmRNAs. The lncRNA-miRNA-mRNA ceRNA network was formed on the basis of the DEmiRNA-DElncRNA and DEmiRNA-DEmRNA interactions, and further visualized using Cytoscape software (version 3.7.2; https://cytoscape. org). A flow diagram of the IncRNA-miRNA-mRNA ceRNA network analysis is shown in Fig. 1.

Survival analysis. Using the median expression of the differentially expressed RNAs as the cutoff value, patients with GC were divided into high and low expression groups. The 


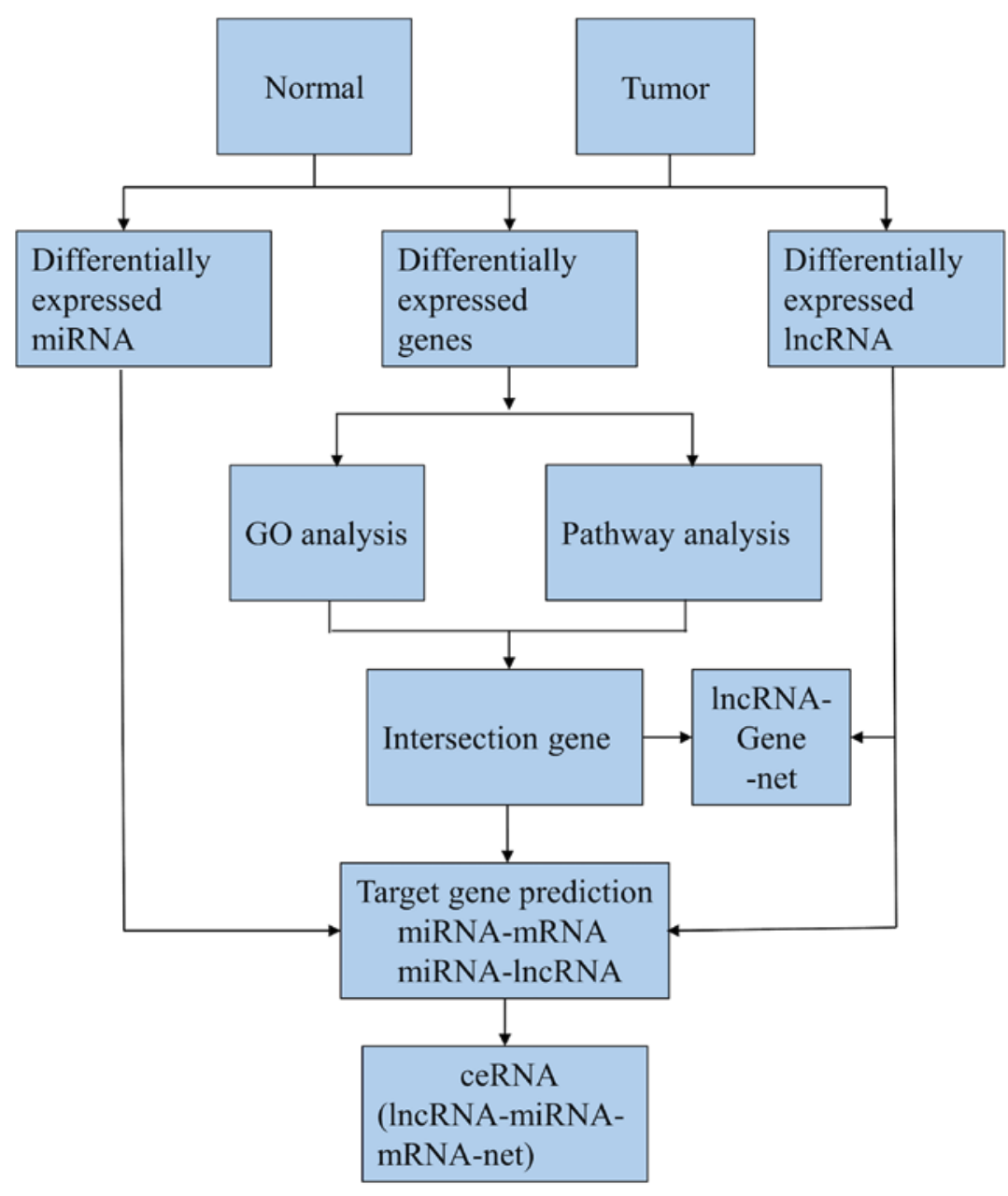

Figure 1. Flow chart of bioinformatics analysis.

difference in survival time between the two groups was evaluated by Kaplan-Meier survival curve and log-rank test analysis via the SPSS Statistics software, version 21 (IBM Corp.). As a result, lncRNAs were found accordingly. $\mathrm{P}<0.05$ was used as a threshold for statistical significance.

\section{Results}

Differential expression of mRNAs, lncRNAs and miRNAs in $G C$. Based on the cutoff threshold, a total of 1,370 DEmRNAs (627 upregulated and 743 downregulated RNAs) was selected from both GC and normal tissues. A total of 393 DElncRNAs (197 upregulated and 196 downregulated RNAs) and 46 DEmiRNAs (24 upregulated and 22 downregulated RNAs) were differentially expressed in GC compared with normal tissues. Unsupervised hierarchical clustering analysis was achieved for all selected DEmRNAs, DElncRNAs and DEmiRNAs. A heatmap was constructed to display the distinctive pattern of RNAs expressed in GC compared with non-tumor samples (Fig. 2A-C). Thereafter, all differentially expressed RNAs were used for further analyses.

GO and KEGG pathway analysis. GO and KEGG pathway analysis uncovered 25 upregulated and downregulated GO and KEGG terms. The most significant GO terms were associated with 'cell division', 'proliferation' and 'mitotic cell cycle' (Fig. 3A and B).
KEGG pathway enrichment analysis was also performed using the selected DEmRNAs. The leading 25 upregulated and downregulated KEGG pathways were found to be permanently enriched by DEmRNAs in the ceRNA network, among which the 'PI3K-Akt signaling pathway', 'cell cycle', 'pathways in cancer' and the 'p53 signaling pathway' were most closely associated with the pathogenesis of cancer (Fig. 3C and D).

Construction of lncRNA-mRNA co-expression network. Subsequently, an lncRNA-mRNA co-expression network was established using the GO terms and pathways compiled by respective target genes and DElncRNAs. The statistical basis of the co-expression network was Pearson correlation index $|r|>0.9$ in a network where both 1 c RNA-mRNA and mRNA-mRNA connections were mutually regulated. The co-expression network in GC and normal gastric tissues was constructed according to the Pearson's correlation coefficient, which measured values of lncRNA and mRNA. Based on preceding conclusions (Figs. 2A and 3), the lncRNA-mRNA co-expression network was created among differentially expressed lncRNAs and mRNAs in both GC and normal gastric tissues. The results revealed network associations between 12 lncRNAs and 57 mRNAs (Fig. S1).

DElncRNA-DEmiRNA interactions predicted by miRcode. The prediction analysis of lncRNA binding to DEmiRNAs was 
A DElncRNAs

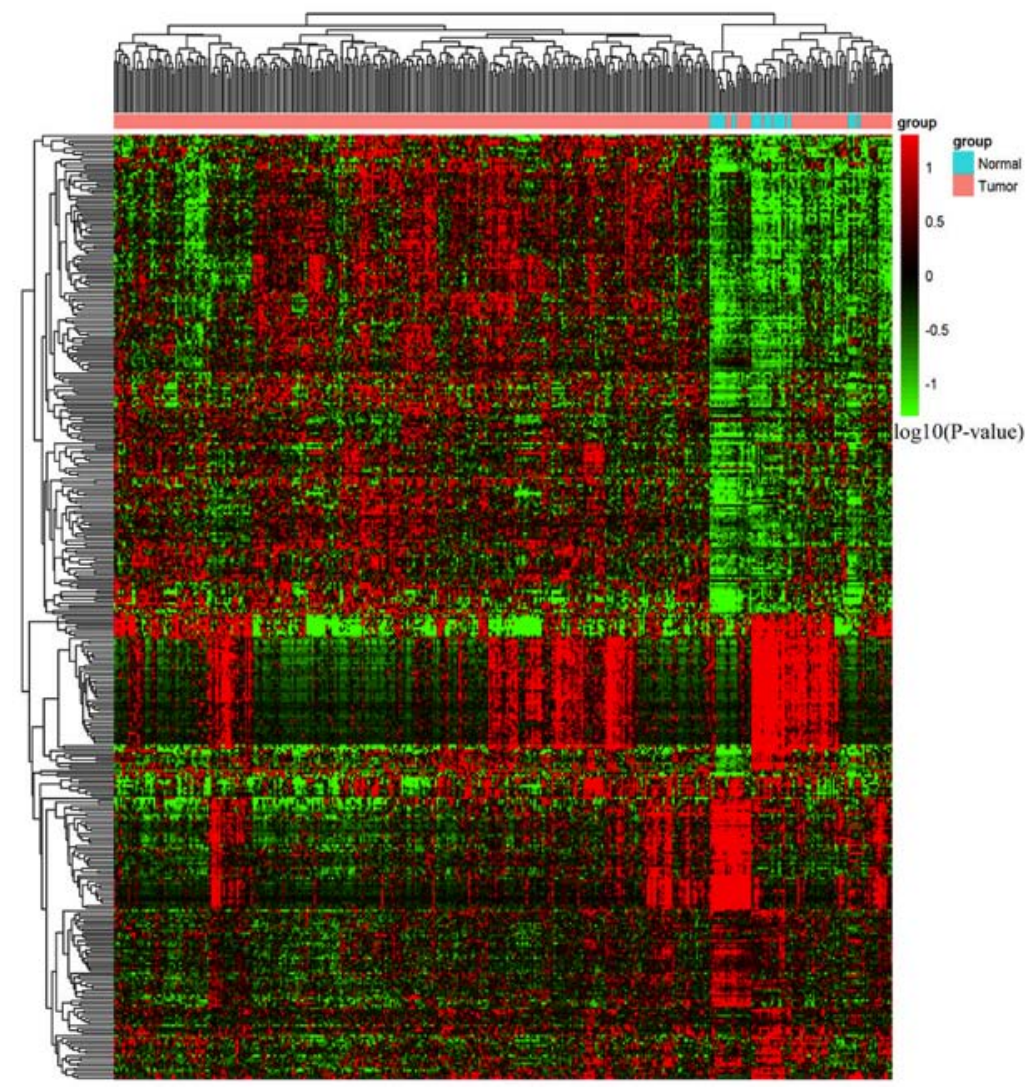

B

DEmiRNAs

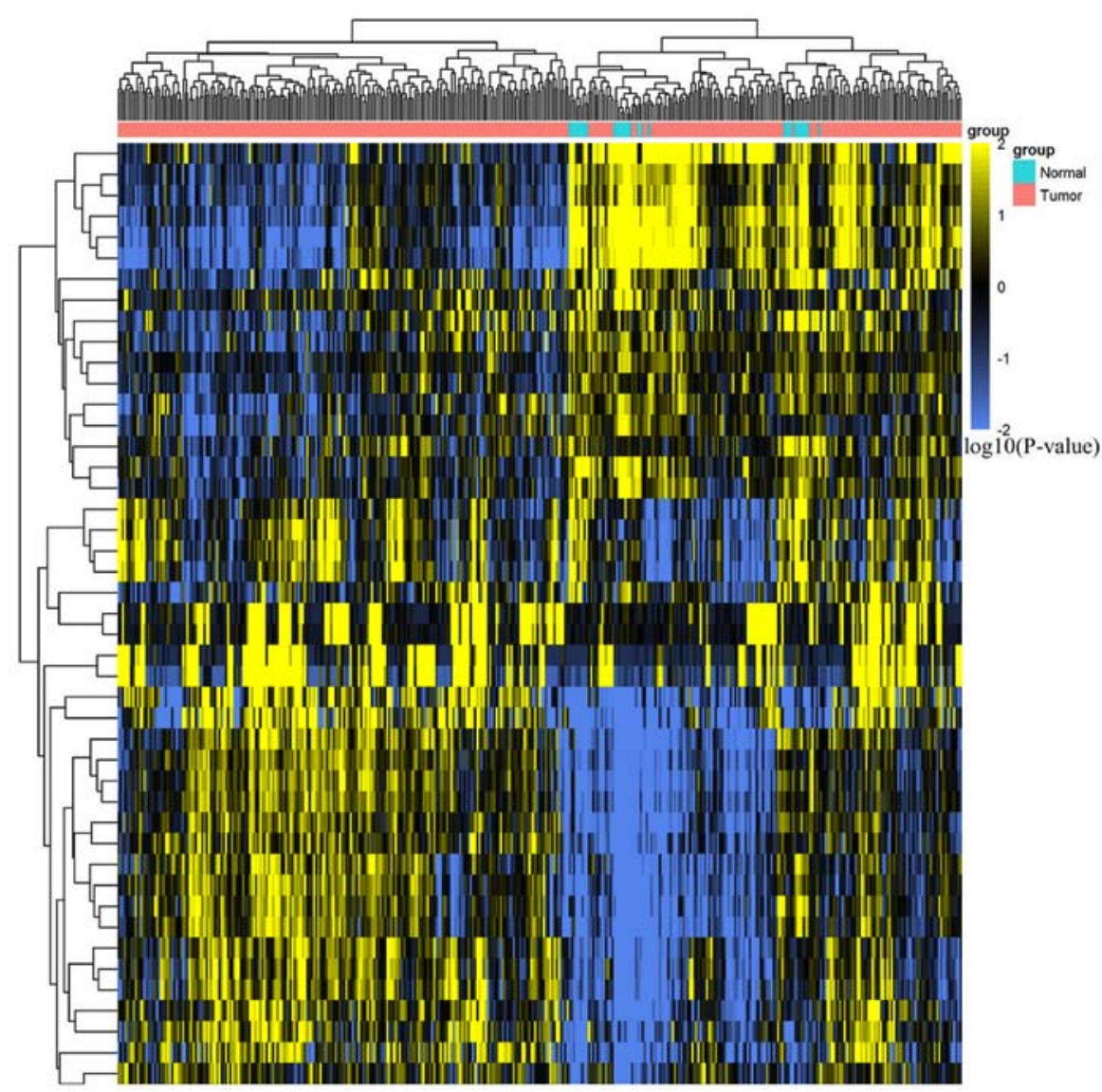

Figure 2. Differential expression of intersecting RNAs in GC. (A) DElncRNAs and (B) DEmiRNAs. A heatmap showing the differentially expressed RNAs. 


\section{DEmRNAs}

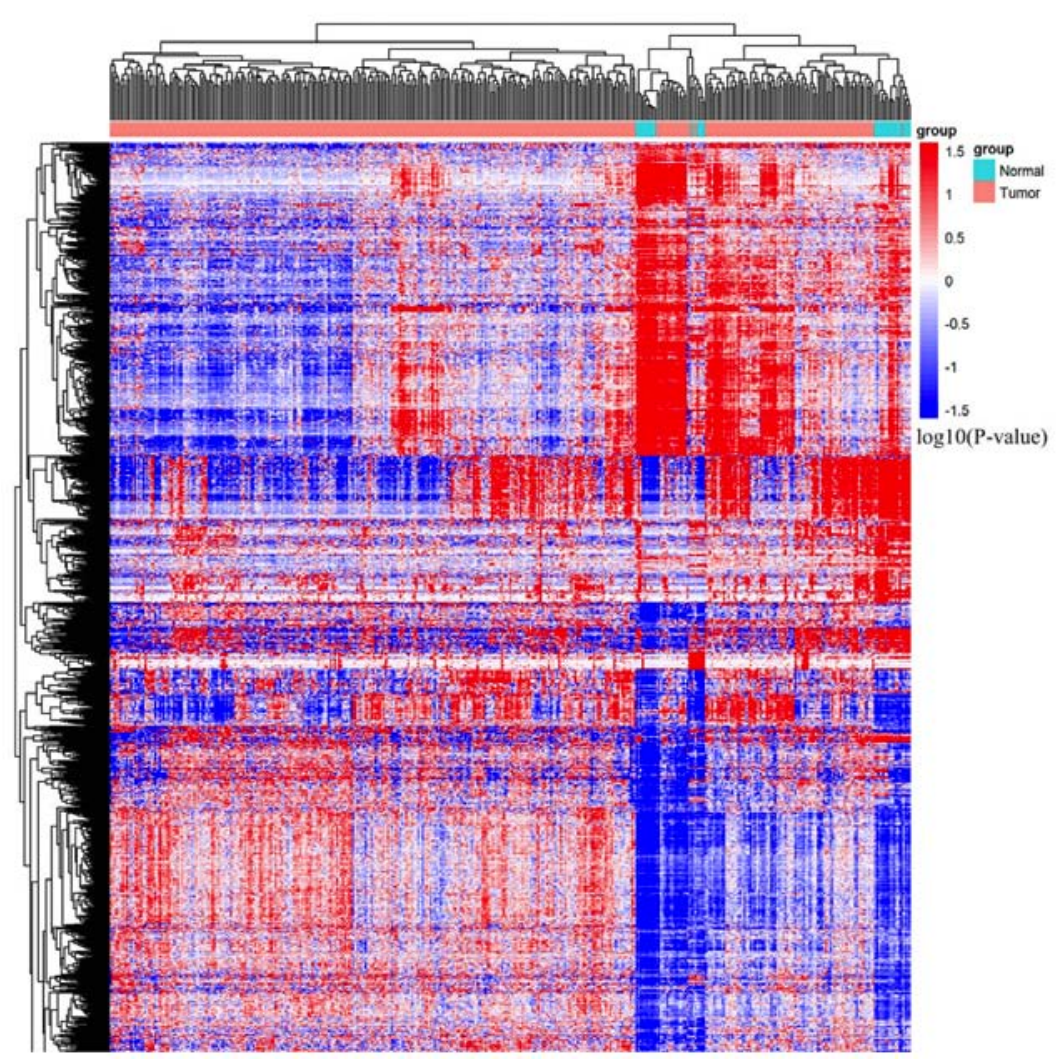

Figure 2. Continued. Differential expression of intersecting RNAs in GC. (C) DEmRNAs. A heatmap showing the differentially expressed RNAs. DE, differentially expressed; IncRNA, long non-coding RNA; miRNA, microRNA.

performed using miRcode. As such, predicted lncRNAs and DElncRNAs were intersected, and the intersecting lncRNAs were included into the ceRNA network. Correspondingly, 342 interactions between 64 DElncRNAs (including PVT1, RP11-284F21.7, RP11-284F21.10, HAND2-AS1 and ZNF667-AS1) and 35 DEmiRNAs (including hsa-miR-194-3p, hsa-miR-196b-5p, hsa-miR-218-5p and hsa-miR-145-3p) were annotated and further analyzed. The top 10 miRNA-mediated lncRNAs are shown in Table II.

Prediction of DEmiRNA-interacting DEmRNAs. Previously annotated DEmiRNAs were mapped into TargetScan, miRTarBase and miRDB databases to search for their target mRNAs. A total of 357 mRNAs were converged by these DEmiRNAs in all three databases. As a result, 118 mRNAs were determined as the DEmiRNA-targeted DEmRNAs. To underline the ceRNA characteristics of the network, DEmiRNAs that were capable of combining with both DEmRNAs and DElncRNAs were introduced into the ceRNA network. Ultimately, 37 DEmiRNAs, 118 DEmRNAs and 65 DElncRNAs were selected for ceRNA network construction. The top 27 miRNAs, which were predicted to modulate most of the mRNAs, are shown in Table III.

Profile of the ceRNA network in GC. Based on data from Tables II and III, and Fig. S1, an miRNA-lncRNA-mRNA ceRNA network was constructed.ThemiRNA-lncRNA-mRNA associations were integrated into the ceRNA network, according to their mutual regulation roles. The ceRNA network was constructed with 220 modules (including 37 DEmiRNAs, 118 DEmRNAs and 65 DElncRNAs) and 717 interactions. The network was profiled using Cytoscape (Fig. 4A and B). This network demonstrated the possibility of indirect associations between DElncRNAs and DEmRNAs.

IncRNAs associated with the prognosis of patients with GC. In order to discover lncRNAs that are significantly correlated with the prognosis of patients with GC, Kaplan-Meier survival curves and log-rank test analyses were conducted for each of the DElncRNAs comprising the ceRNA network. The results demonstrated that three distinct DElncRNAs (PVT1, ZNF667-AS1 and HAND2-AS1) were associated with survival (P<0.05; Fig. 5A-C). ZNF667-AS1 and HAND2-AS1 appeared to be pro-cancerous, since patients with higher levels of these RNAs presented with shorter overall survival time (Fig. 5A and C). By contrast, PVT1 appeared to have a protective role, since its high expression was associated with longer overall survival time in patients with GC (Fig. 5C).

\section{Discussion}

GC is one of the most common types of cancer worldwide, and it largely affects the quality of life and the health of Asian populations. In China, the incidence and mortality rates of GC have escalated to be the second highest among all cancer types (20). Although the diagnosis and treatment of GC have significantly 
Table II. Top 10 DElncRNAs putatively targeted by most DEmiRNAs in the competing endogenous RNA network.

DElncRNAs

\section{DEmiRNAs}

MIR143HG hsa-miR-141-5p, hsa-miR-146b-5p, hsa-miR-18a-5p, hsa-miR-196a-5p, hsa-miR-196b-5p, hsa-miR-335-3p, hsa-miR-552-5p, hsa-miR-767-5p

LINC00261 hsa-miR-105-5p, hsa-miR-135b-5p, hsa-miR-182-5p, hsa-miR-194-3p, hsa-miR-196a-5p, hsa-miR-335-3p, hsa-miR-552-3p

TP73-AS1

GS1-358P8.4

MBNL1-AS1

AFAP1-AS1

NSUN5P1 hsa-miR-105-5p, hsa-miR-141-3p, hsa-miR-141-5p, hsa-miR-194-3p, hsa-miR-552-3p

RP11-242D8.1 hsa-miR-141-3p, hsa-miR-146b-5p, hsa-miR-196a-5p, hsa-miR-196b-5p, hsa-miR-200a-3p

MIR4435-2HG

TUG1 hsa-miR-141-3p, hsa-miR-196a-5p, hsa-miR-200a-3p, hsa-miR-767-5p hsa-miR-129-5p, hsa-miR-145-3p, hsa-miR-149-5p, hsa-miR-30c-2-3p hsa-miR-129-5p, hsa-miR-139-3p, hsa-miR-30c-2-3p, hsa-miR-5683 hsa-miR-145-3p, hsa-miR-149-5p, hsa-miR-30c-2-3p hsa-miR-1-3p, hsa-miR-145-5p, hsa-miR-149-5p hsa-miR-144-3p, hsa-miR-29c-3p, hsa-miR-5683

DE, differentially expressed; lncRNA, long non-coding RNA; miRNA, microRNA.

A

Top 25 upregulated GO genes

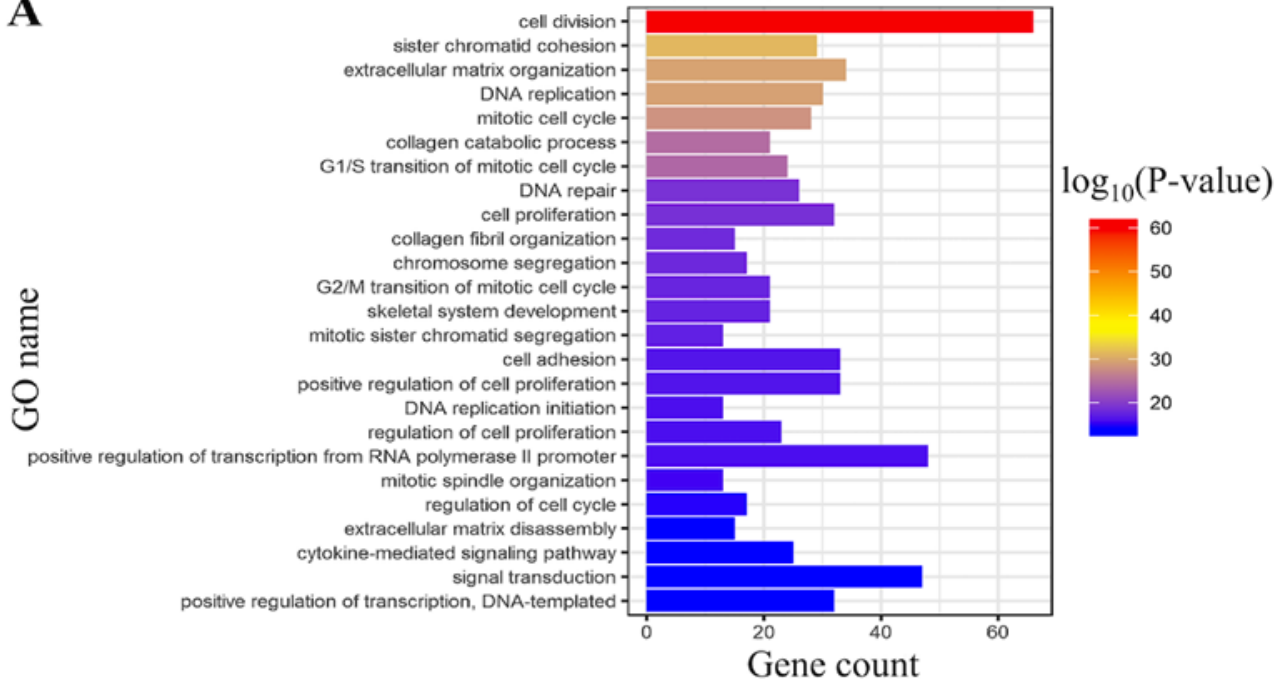

B

Top 25 downregulated GO genes

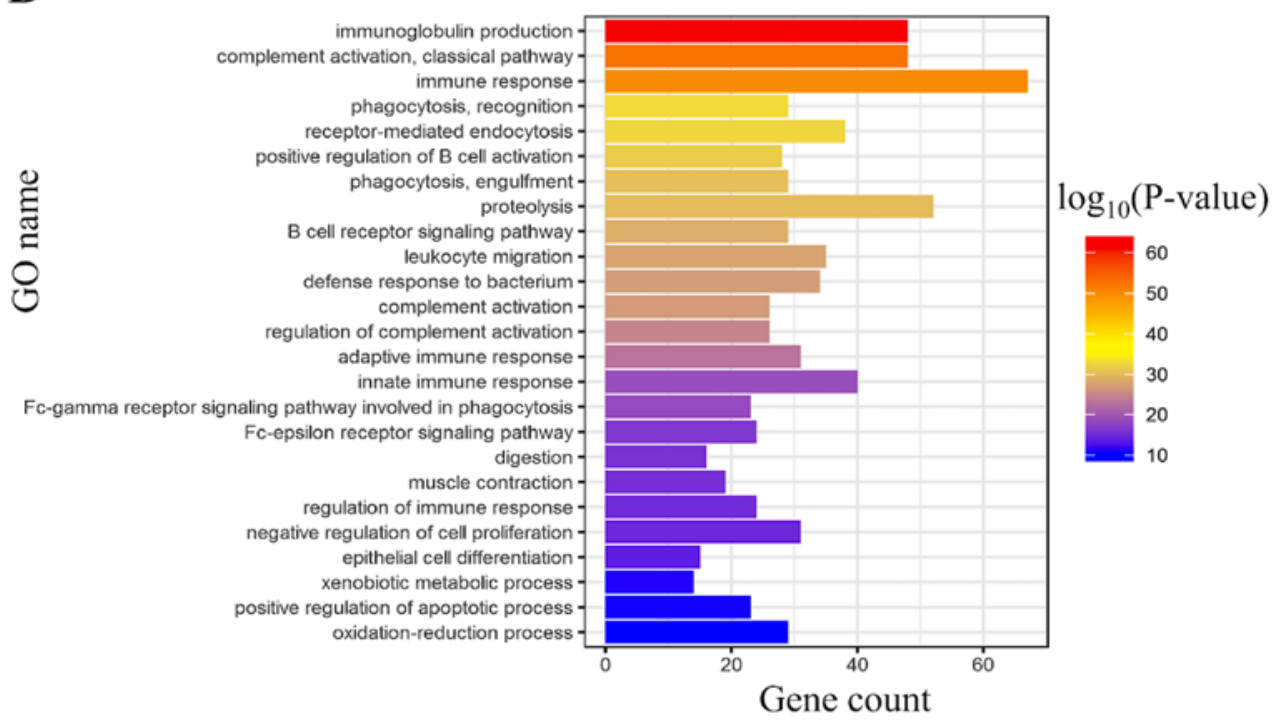

Figure 3. Top 25 enrichment GO terms and pathways of differentially expressed intersecting mRNAs. (A) Upregulated GO terms, and (B) downregulated GO terms. 


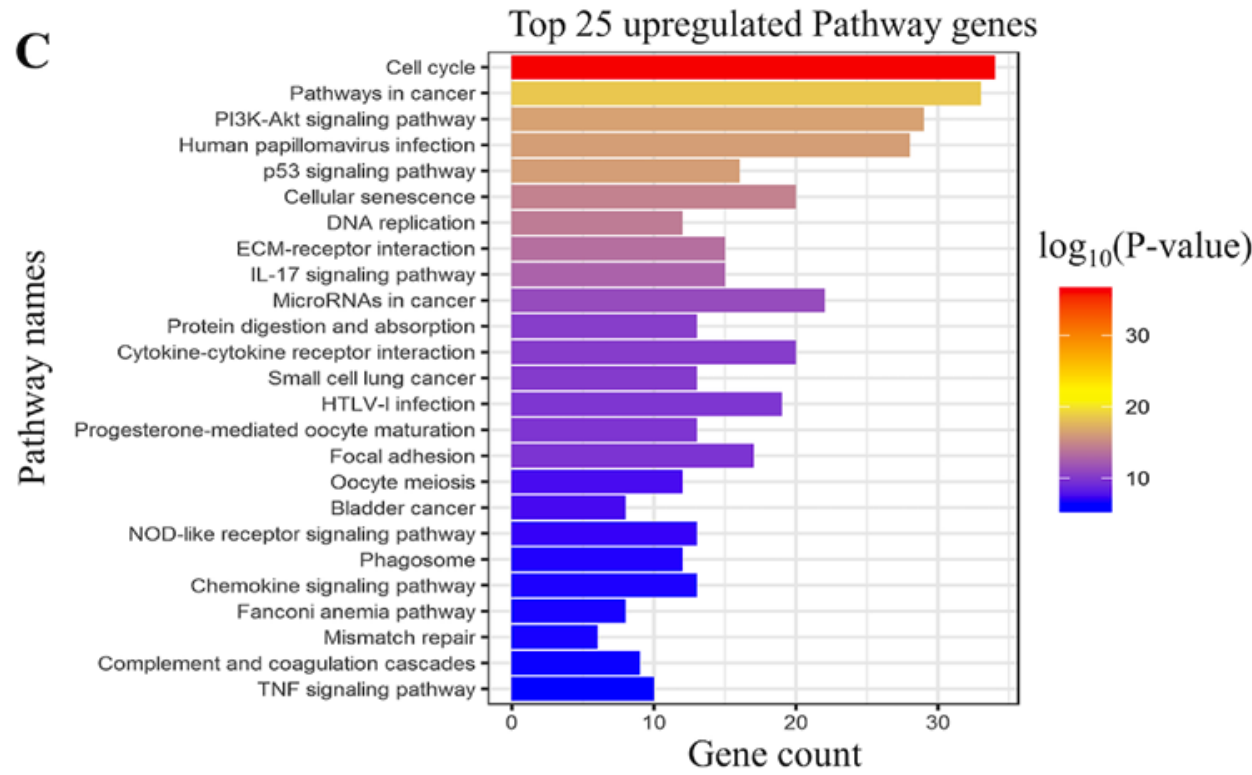

D

Top 25 downregulated Pathway genes

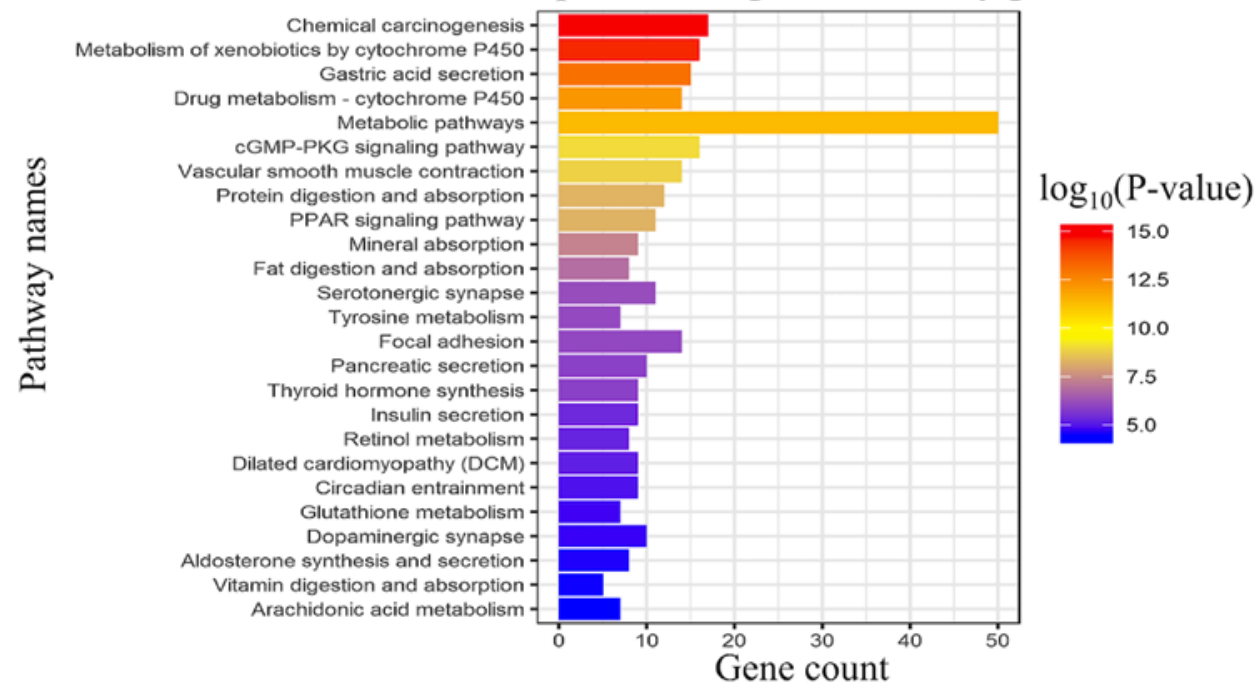

Figure 3. Continued. Top 25 enrichment GO terms and pathways of differentially expressed intersecting mRNAs. (C) upregulated pathways and (D) downregulated pathways. The bar plot shows the enrichment scores of the significant enrichment GO terms and pathways. GO, Gene Ontology.

improved, the 5-year overall survival rate of patients with advanced GC is still $\sim 20 \%$, mostly due to late diagnosis, rapid progression and metastatic characteristics $(21,22)$. Therefore, the discovery and characterization of early GC biomarkers is imperative for a more efficient diagnosis and treatment of the disease.

The role of lncRNAs in cancer has become increasingly important. Moreover, $\operatorname{lncRNAs}$ appear to play a vital role in cancer proliferation, development and prognosis. For example, MEG3 inhibits GC metastasis by activating the p53 signaling pathway (23). The IncRNA AWPPH enhances the growth and proliferation of triple-negative breast cancer by upregulating FZD7 (24), whereas cancer progression and shortened survival in patients with colon cancer are associated with the overexpression of the IncRNA DANCR (25).

A number of studies have shown that IncRNAs can regulate diverse biological activities in tumor cells, including transcriptional, post-transcriptional and phenotype regulation (26-28). IncRNAs influence the stability of target-encoding genes by modulating the activity of miRNAs (29). In the context of GC, a complicated regulatory network involving lncRNAs and miRNAs or mRNAs plays a vital role in the occurrence and development of the disease $(30,31)$. Therefore, the concept of ceRNAs was proposed in order to explain the mutual regulatory mechanism between non-coding and coding RNAs during cancer progression, as well as its influence on the survival of patients with cancer (11). Compared with the coding RNAs, lncRNAs have a greater advantage as an effective biomarker for the diagnosis and prognosis of early GC (32).

A number of lncRNAs have been found to be associated with GC. It is also well known that Helicobacter pylori infection plays a role in the pathogenesis of GC. In vitro and in vivo studies have revealed that infection by $\mathrm{H}$. pylori can, for example, drive the downregulation of IncRNA AF147447 in GC. IncRNA AF147447 can suppress the proliferation and invasion of GC by repressing MU2 and upregulating the expression of miR-34c (33). The IncRNA NORAD is highly 
A

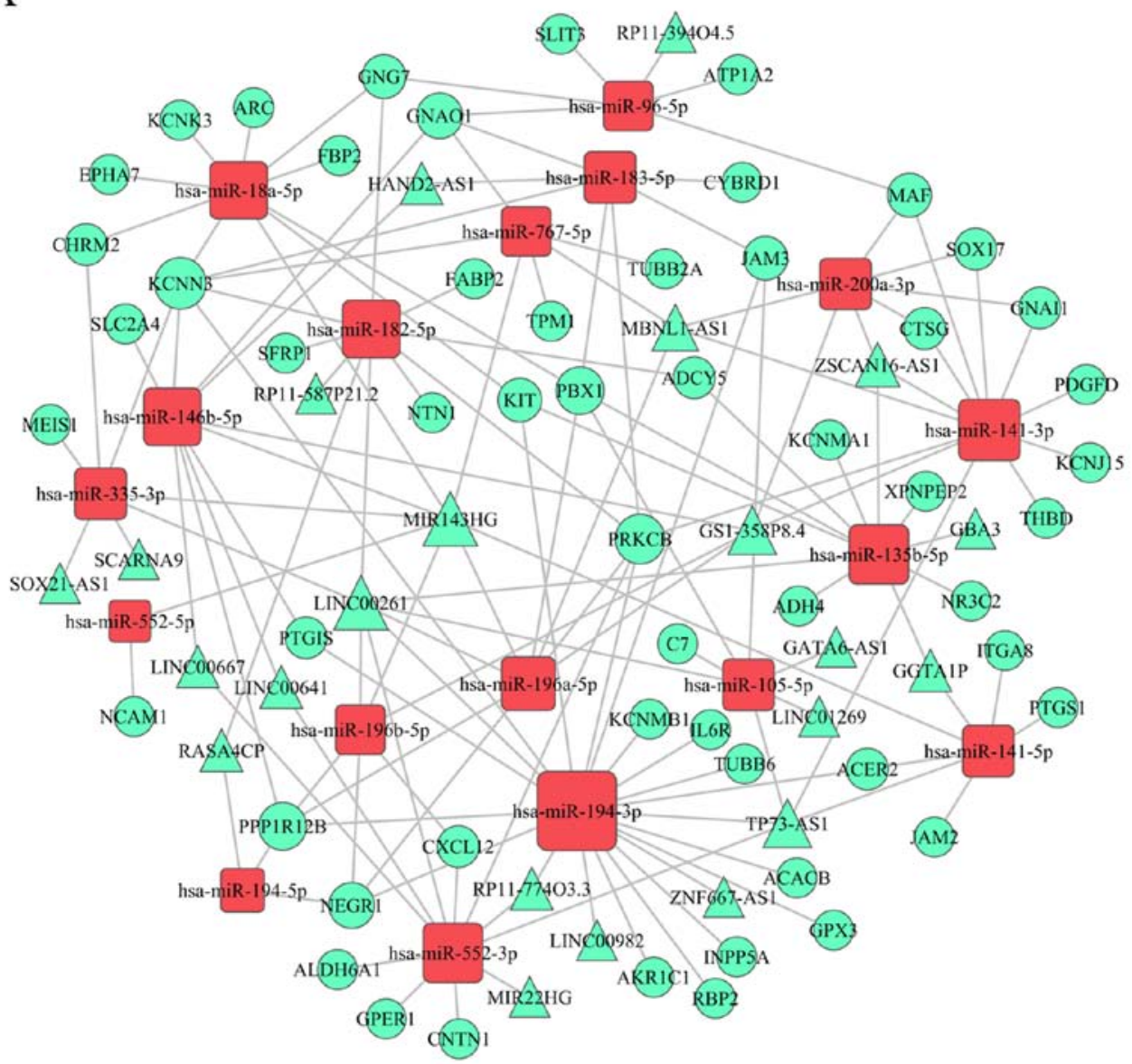

B

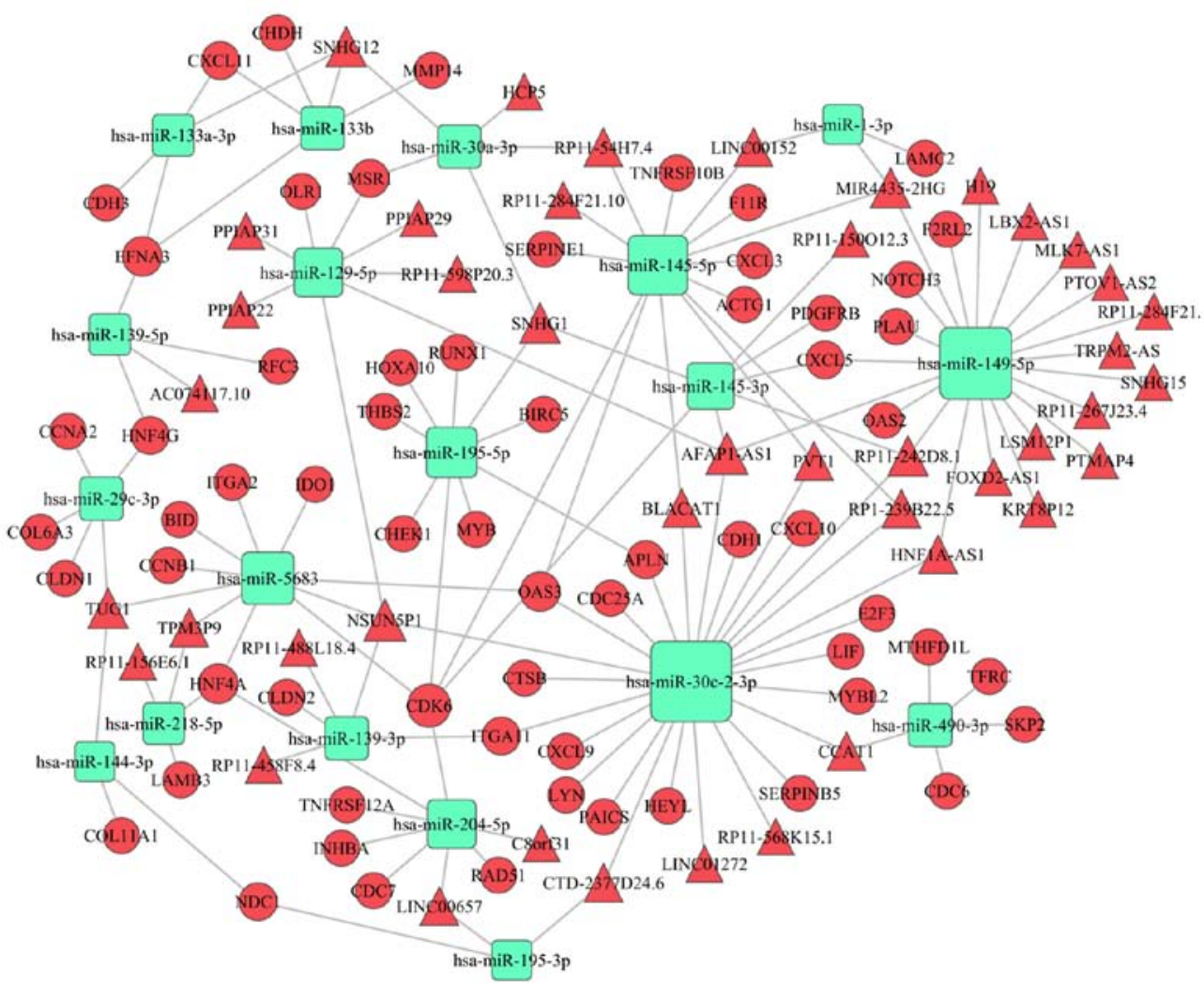

Figure 4. IncRNA-miRNA-mRNA competing endogenous RNA network. (A) Red squares represent upregulated miRNAs, green circles represent downregulated mRNAs and green triangles represent downregulated lncRNAs. (B) Green squares represent downregulated miRNAs, red circles represent upregulated mRNAs and red triangles represent upregulated lncRNAs. miRNA/miR, microRNA; lncRNA, long non-coding RNA. 
Table III. DEmiRNAs (n=27) with corresponding target DEmRNAs in the competing endogenous RNA network.

\begin{tabular}{|c|c|}
\hline DEmiRNAs & DEmRNAs \\
\hline hsa-miR-194-3p & $\begin{array}{l}\text { ACACB, ACER2, AKR1C1, GPX3, IL6R, INPP5A, JAM3, KCNMB1, KCNN3, KIT, NEGR1, PPP1R12B, } \\
\text { PRKCB, PTGIS, RBP2, TUBB6 }\end{array}$ \\
\hline hsa-miR-30c-2-3p & $\begin{array}{l}\text { LYN, APLN, CDC25A, CDH1, CTSB, CXCL10, CXCL9, E2F3, HEYL, ITGA11, LIF, MYBL2, OAS3, } \\
\text { PAICS, SERPINB5 }\end{array}$ \\
\hline hsa-miR-195-5p & APLN, BIRC5, CDK6, CHEK1, HOXA10, MYB, RUNX1, THBS2 \\
\hline hsa-miR-141-3p & CTSG, GNAI1, KCNJ15, MAF, PDGFD, PRKCB, SOX17, THBD \\
\hline hsa-miR-145-5p & ACTG1, CDK6, CXCL3, F11R, OAS3, SERPINE1, TNFRSF10B \\
\hline hsa-miR-135b-5p & ADCY5, ADH4, KCNMA1, KIT, NR3C2, PBX1, XPNPEP2 \\
\hline hsa-miR-18a-5p & ARC, CHRM2, EPHA7, FBP2, GNG7, KCNK3, KIT, PBX1 \\
\hline hsa-miR-182-5p & ADCY5, FABP2, GNG7, KCNN3, NTN1, PRKCB, SFRP1 \\
\hline hsa-miR-183-5p & CYBRD1, GNAO1, JAM3, KCNN3, PBX1, PRKCB \\
\hline hsa-miR-5683 & BID, CCNB1, CDK6, HNF4A, IDO1, ITGA2, OAS3 \\
\hline hsa-miR-552-3p & ALDH6A1, CNTN1, CXCL12, GPER1, PRKCB \\
\hline hsa-miR-146b-5p & GNAO1, KCNN3, PPP1R12B, PTGIS, SLC2A4 \\
\hline hsa-miR-204-5p & CDC7, CDK6, INHBA, RAD51, TNFRSF12A \\
\hline hsa-miR-149-5p & CXCL5, F2RL2, NOTCH3, OAS2, PLAU \\
\hline hsa-miR-96-5p & ATP1A2, GNAO1, GNG7, MAF, SLIT3 \\
\hline hsa-miR-196a-5p & NEGR1, PBX1, PPP1R12B, PRKCB \\
\hline hsa-miR-29c-3p & CCNA2, CLDN1, COL6A3, HNF4G \\
\hline hsa-miR-767-5p & GNAO1, KCNN3, TPM1, TUBB2A \\
\hline hsa-miR-133b & CHDH, CXCL11, EFNA3, MMP14 \\
\hline hsa-miR-490-3p & CDC6, MTHFD1L, SKP2, TFRC \\
\hline hsa-miR-141-5p & ACER2, ITGA8, JAM2, PTGS1 \\
\hline hsa-miR-196b-5p & CXCL12, NEGR1, PPP1R12B \\
\hline hsa-miR-200a-3p & CTSG, GNAI1, MAF, SOX17 \\
\hline hsa-miR-335-3p & CHRM2, KCNN3, MEIS1 \\
\hline hsa-miR-133a-3p & CDH3, CXCL11, EFNA3 \\
\hline hsa-miR-139-5p & EFNA3, HNF4G, RFC3 \\
\hline hsa-miR-144-3p & COL11A1, NDC1 \\
\hline
\end{tabular}

DE, differentially expressed; miRNA, microRNA.

expressed in GC tissues and cells, and is strongly correlated with poor clinical outcomes in GC. Furthermore, NORAD may act as a ceRNA to promote the outgrowth of GC cells, by regulating the miR-608/FOXO6 signaling cascade (34). The lncRNA LINC00460 has also been suggested as an independent risk factor for the prognosis of GC, since it can promote the proliferation and migration of cancer cells by stimulating the Wnt/ $\beta$-catenin pathway (35). In addition, the lncRNA FLVCR1-AS1 sponges miR-155 to further promote the development of GC (36).

Following TCGA analysis of the DElncRNA content in GC and paracancerous tissue samples, MIR100HG was found to be highly expressed in GC and, thus, could be considered as a potential prognostic marker of GC (37). AKT protein kinase has been widely studied as a cancer-promoting factor. A recent study reported that lncRNA STXBP5-AS1 can inhibit the activation of AKT in GC, which leads to the inhibition of proliferation and migration of GC cells (38).

The present study attempted to identify a highly specific lncRNA in GC, and to further understand its impact during the pathogenesis and progression of the disease. In order to achieve this aim, the expression profiles of lncRNAs, miRNAs and mRNAs were first assessed in $361 \mathrm{GC}$ and 32 adjacent and normal tissues from TCGA database. A detailed bioinformatics analysis of GC compared with normal tissues uncovered some DElncRNAs (PVT1, RP11-284F21.7 and RP11-284F21.10) that were significantly upregulated, and at least two DElncRNAs (HAND2-AS1 and ZNF667-AS1) that were significantly downregulated. Moreover, a number of DEmiRNAs and DEmRNAs were upregulated and downregulated. In particular, enriched DEmRNAs were closely associated to processes that impact carcinogenesis, including 'cell division', 'proliferation' and the 'mitotic cell cycle'. Moreover, a number of DEmRNAs were associated with the 'PI3K-Akt signaling pathway' (39), 'pathways in cancer' and the 'p53 signaling pathway' (40) in GC, and abnormalities in these pathways are also associated with cancer. Subsequently, the association between the three RNAs was analyzed. Furthermore, a ceRNA network map was constructed, and Kaplan-Meier survival analyses were performed to establish a potential association between differential RNAs and 
$\mathbf{A}$

HAND2-AS1, $P=0.046$

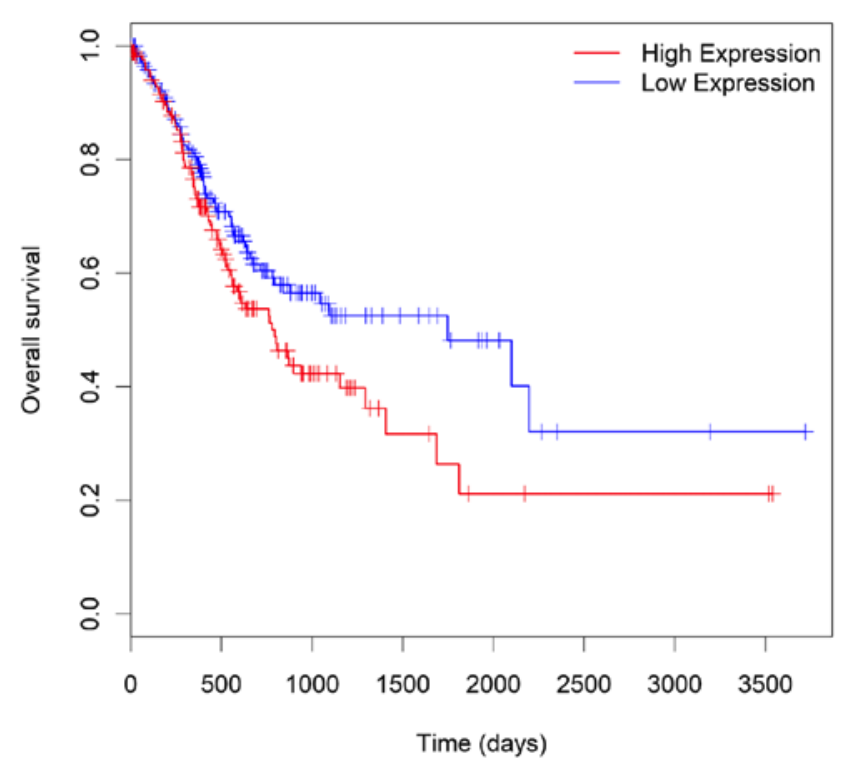

B

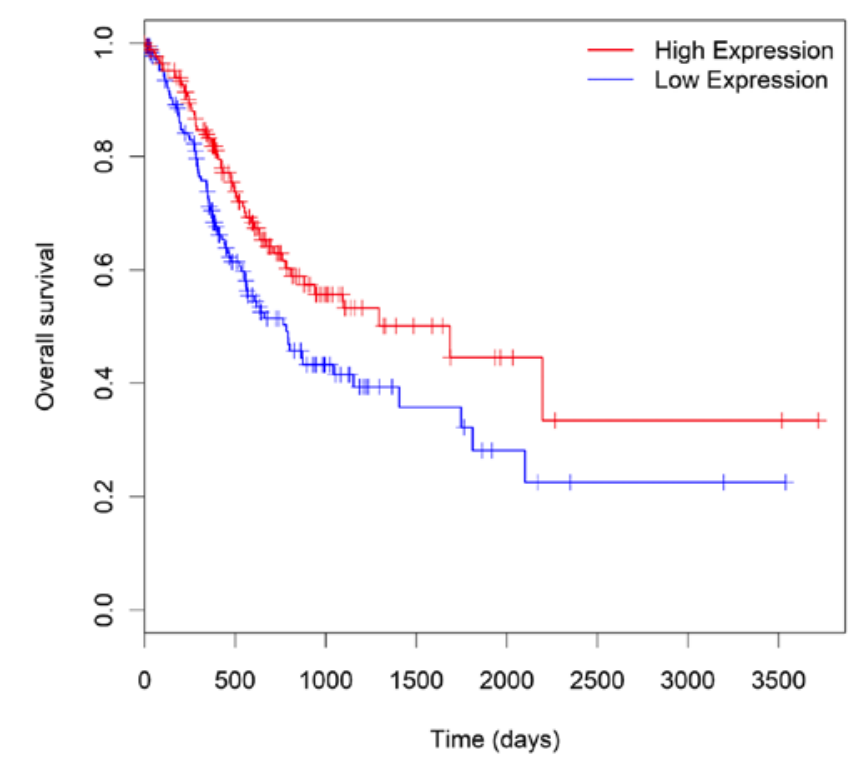

C

ZNF667-AS1, $P=0.0167$

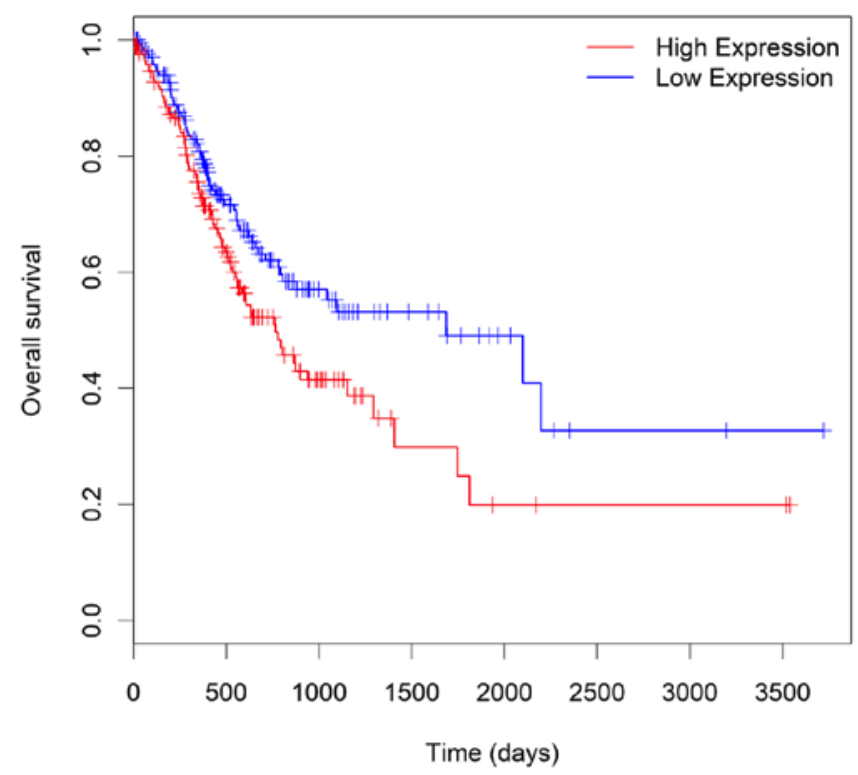

Figure 5. Kaplan-Meier survival curves of the three long non-coding RNAs. Kaplan-Meier survival curves of patients with low and high expression levels of (A) HAND2-AS1, (B) PVT1 and (C) ZNF667-AS1.

the prognosis of GC. The lncRNAs HAND2-AS1, PVT1 and ZNF667-AS1 were strongly associated with the prognosis of GC and could be used as biomarkers for predicting the prognosis of the disease.

The role of PVT1 in other cancer types has been previously reported. For instance, it was shown that PVT1 was highly expressed in patients with glioma at stages III-IV (versus patients at stages I-II), and the survival time of these patients with high expression of PVT1 was significantly shorter (41). PVT1 is also involved in the sensitivity of radiotherapy affecting lung cancer (42). The role of IncRNA HAND2-AS1, which was also reported in the present study, has been similarly identified in other cancer types. As an example, HAND2-AS1 appears to inhibit the proliferation and migration of esophageal squamous cell tumors (43). HAND2-AS1 can also impede the growth and metastasis of colon cancer by upregulating KLF5 (44). In lung cancer, HAND2-AS1 can also act as a tumor suppressor (45). However, the roles of ZNF667-AS1 and HAND2-AS1 in GC has not been previously reported. To the best of our knowledge, the present study is the first to demonstrate that high expression of ZNF667-AS1 and HAND2-AS1 is negatively associated with the survival time of patients with GC.

The construction of an IncRNA-miRNA-mRNA ceRNA network revealed the associations between novel specific IncRNAs and other RNAs in GC. Moreover, a putative association between differentially expressed RNAs and the 
prognosis of patients with GC was elucidated. The findings of the present study may have substantial clinical significance; however, some limitations should be taken into consideration. Firstly, the data were downloaded from TCGA databases; thus, experimental methods are required to verify the results. Secondly, the role of HAND2-AS1, PVT1 and ZNF667-AS1 in GC is still unknown; therefore, in vivo and in vitro experiments are required to answer this question.

In conclusion, the data from the present study will serve as a basis for the definition and validation of potential biomarkers for the diagnosis and targeted therapy for GC. Future functional investigations are required to explore the mechanisms underlying the roles of these lncRNAs in GC.

\section{Acknowledgements}

Not applicable.

\section{Funding}

The present study was funded by the Chinese National Natural Science Foundation (grant no. 81871995) and The Special Funding for Doctoral Team of the First Affiliated Hospital of Zhengzhou University (grant no. 2016-BSTDJJ-11).

\section{Availability of data and materials}

The datasets used and/or analyzed during the present study are available from the corresponding author upon reasonable request.

\section{Authors' contributions}

YF and SP conceived and designed the present study, SP drafted the manuscript and YF revised the initial manuscript. XY acquired the data. SP, XY, YZ, WM, TL, YY, JJ and QL analyzed and interpreted the data. All authors have read and approved the final manuscript.

\section{Ethics approval and consent to participate}

Not applicable.

\section{Patient consent for publication}

Not applicable.

\section{Competing interests}

The authors declare that they have no competing interests.

\section{References}

1. Torre LA, Bray F, Siegel RL, Ferlay J, Lortet-Tieulent J and Jemal A: Global cancer statistics, 2012. CA Cancer J Clin 65: 87-108, 2015

2. Sun Z, Wang ZN, Zhu Z, Xu YY, Xu Y, Huang BJ, Zhu GL and $\mathrm{Xu}$ HM: Evaluation of the seventh edition of American joint committee on cancer TNM staging system for gastric cancer: Results from a Chinese monoinstitutional study. Ann Surg Oncol 19: 1918-1927, 2012.

3. Hombach S and Kretz M: Non-coding RNAs: Classification, biology and functioning. Adv Exp Med Biol 937: 3-17, 2016.
4. Mercer TR, Dinger ME and Mattick JS: Long non-coding RNAs: Insights into functions. Nat Rev Genet 10: 155-159, 2009.

5. He Y, Meng XM, Huang C, Wu BM, Zhang L, Lv XW and Li J: Long noncoding RNAs: Novel insights into hepatocelluar carcinoma. Cancer Lett 344: 20-27, 2014.

6. Fatica A and Bozzoni I: Long non-coding RNAs: New players in cell differentiation and development. Nat Rev Genet 15: 7-21, 2014.

7. $\mathrm{Li} \mathrm{CH}$ and Chen $\mathrm{Y}$ : Insight into the role of long noncoding RNA in cancer development and progression. Int Rev Cell Mol Biol 326: 33-65, 2016.

8. Kino T, Hurt DE, Ichijo T, Nader N and Chrousos GP: Noncoding RNA gas 5 is a growth arrest- and starvation-associated repressor of the glucocorticoid receptor. Sci Signal 3: ra8, 2010.

9. Bartel DP: MicroRNAs: Genomics, biogenesis, mechanism, and function. Cell 116: 281-297, 2004.

10. Bartel DP: MicroRNAs: Target recognition and regulatory functions. Cell 136: 215-233, 2009.

11. Cai Y, Yu X, Hu S and Yu J: A brief review on the mechanisms of miRNA regulation. Genomics Proteomics Bioinformatics 7: 147-154, 2009.

12. Salmena L, Poliseno L, Tay Y, Kats L and Pandolfi PP: A ceRNA hypothesis: The Rosetta Stone of a hidden RNA language? Cell 146: 353-358, 2011

13. Chan JJ and Tay Y: Noncoding RNA:RNA regulatory networks in cancer. Int J Mol Sci 19: E1310, 2018.

14. Li H, Yu B, Li J, Su L, Yan M, Zhu Z and Liu B: Overexpression of lncRNA H19 enhances carcinogenesis and metastasis of gastric cancer. Oncotarget 5: 2318-2329, 2014.

15. Wu X, Zhang P, Zhu H, Li S, Chen X and Shi L: Long noncoding RNA FEZF1-AS1 indicates a poor prognosis of gastric cancer and promotes tumorigenesis via activation of Wnt signaling pathway. Biomed Pharmacother 96: 1103-1108, 2017.

16. Chen QN, Wei CC, Wang ZX and Sun M: Long non-coding RNAs in anti-cancer drug resistance. Oncotarget 8: 1925-1936, 2017.

17. Goldman M, Craft B, Swatloski T, Cline M, Morozova O, Diekhans M, Haussler D and Zhu J: The UCSC cancer genomics browser: Update 2015. Nucleic Acids Res 43: D812-D817, 2015.

18. Anders $\mathrm{S}$ and Huber W: Differential expression analysis for sequence count data. Genome Biol 11: R106, 2010.

19. Kimes PK, Liu Y, Neil Hayes D and Marron JS: Statistical significance for hierarchical clustering. Biometrics 73: 811-821, 2017.

20. Chen W, Zheng R, Baade PD, Zhang S, Zeng H, Bray F, Jemal A, Yu XQ and He J: Cancer statistics in China, 2015. CA Cancer J Clin 66: 115-132, 2016.

21. Otterstad JE, Forfang K, Vatne K and Frøysaker T: Posterior left ventricular aneurysm due to occlusion of the circumflex coronary artery with recurrent ventricular tachycardia. Case report. Scand J Thorac Cardiovasc Surg 16: 205-208, 1982.

22. Liu H, Dong Y, Gao Y, Du Z, Wang Y, Cheng P, Chen A and Huang $\mathrm{H}$ : The fascinating effects of baicalein on cancer: A review. Int J Mol Sci 17: E1681, 2016.

23. Wei GH and Wang X: lncRNA MEG3 inhibit proliferation and metastasis of gastric cancer via p53 signaling pathway. Eur Rev Med Pharmacol Sci 21: 3850-3856, 2017.

24. Wang K, Li X, Song C and Li M: LncRNA AWPPH promotes the growth of triple-negative breast cancer by up-regulating frizzled homolog 7 (FZD7). Biosci Rep 38: BSR20181223, 2018.

25. Liu Y, Zhang M, Liang L, Li J and Chen YX: Over-expression of lncRNA DANCR is associated with advanced tumor progression and poor prognosis in patients with colorectal cancer. Int J Clin Exp Pathol 8: 11480-11484, 2015.

26. Muers M: RNA: Genome-wide views of long non-coding RNAs. Nat Rev Genet 12: 742, 2011.

27. Kornienko AE, Guenzl PM, Barlow DP and Pauler FM: Gene regulation by the act of long non-coding RNA transcription. BMC Biol 11: 59, 2013.

28. Wahlestedt C: Targeting long non-coding RNA to therapeutically upregulate gene expression. Nat Rev Drug Discov 12: 433-446, 2013.

29. Li T, Mo X, Fu L, Xiao B and Guo J: Molecular mechanisms of long noncoding RNAs on gastric cancer. Oncotarget 7: 8601-8612, 2016

30. Zeng X, Zhang X and Zou Q: Integrative approaches for predicting microRNA function and prioritizing disease-related microRNA using biological interaction networks. Brief Bioinform 17: 193-203, 2016. 
31. Liu Y, Zeng X, He Z and Zou Q: Inferring microRNA-disease associations by random walk on a heterogeneous network with multiple data sources. IEEE/ACM Trans Comput Biol Bioinform 14: 905-915, 2017.

32. Sanchez Calle A, Kawamura Y, Yamamoto Y, Takeshita F and Ochiya T: Emerging roles of long non-coding RNA in cancer. Cancer Sci 109: 2093-2100, 2018.

33. Zhou X, Chen H, Zhu L, Hao B, Zhang W, Hua J, Gu H, Jin W and Zhang G: Helicobacter pylori infection related long noncoding RNA (lncRNA) AF147447 inhibits gastric cancer proliferation and invasion by targeting MUC2 and up-regulating miR-34c. Oncotarget 7: 82770-82782, 2016.

34. Miao Z, Guo X and Tian L: The long noncoding RNA NORAD promotes the growth of gastric cancer cells by sponging miR-608. Gene 687: 116-124, 2019.

35. Zhang S, Xu J, Wang $\mathrm{H}$ and Guo H: Downregulation of long noncoding RNA LINC00460 expression suppresses tumor growth in vitro and in vivo in gastric cancer. Cancer Biomark 24 429-437, 2019.

36. Liu Y, Guo G, Zhong Z, Sun L, Liao L, Wang X, Cao Q and Chen H: Long non-coding RNA FLVCR1-AS1 sponges miR-155 to promote the tumorigenesis of gastric cancer by targeting c-Myc. Am J Transl Res 11: 793-805, 2019.

37. Li J, Xu Q, Wang W and Sun S: MIR100HG: a credible prognostic biomarker and an oncogenic lncRNA in gastric cancer. Biosci Rep 39: BSR20190171, 2019.

38. Cen D, Huang $\mathrm{H}$, Yang L, Guo $\mathrm{K}$ and Zhang J: Long noncoding RNA STXBP5-AS1 inhibits cell proliferation, migration, and invasion through inhibiting the PI3K/AKT signaling pathway in gastric cancer cells. Onco Targets Ther 12: 1929-1936, 2019.
39. Noorolyai S, Shajari N, Baghbani E, Sadreddini S and Baradaran B: The relation between PI3K/AKT signalling pathway and cancer. Gene 698: 120-128, 2019.

40. Joerger AC and Fersht AR: The p53 pathway: Origins, inactivation in cancer, and emerging therapeutic approaches. Annu Rev Biochem 85: 375-404, 2016.

41. Fang $\mathrm{J}$ and Huang J: Clinical significance of the expression of long non-coding RNA PVT1 in glioma. Cancer Biomark 24: 509-513, 2019.

42. Wang D and Hu Y: Long non-coding RNA PVT1 competitively binds microRNA-424-5p to regulate CARM1 in radiosensitivity of non-small-cell lung cancer. Mol Ther Nucleic Acids 16: 130-140, 2018

43. Yan Y, Li S, Wang S, Rubegni P, Tognetti L, Zhang J and Yan L: Long noncoding RNA HAND2-AS1 inhibits cancer cell proliferation, migration, and invasion in esophagus squamous cell carcinoma by regulating microRNA-21. J Cell Biochem 120: 9564-9571, 2019.

44. Zhou J, Lin J, Zhang H, Zhu F and Xie R: LncRNA HAND2-AS1 sponging miR-1275 suppresses colorectal cancer progression by upregulating KLF14. Biochem Biophys Res Commun 503: 1848-1853, 2018.

45. Miao F, Chen J, Shi M, Song Y, Chen Z and Pang L: LncRNA HAND2-AS1 inhibits non-small cell lung cancer migration, invasion and maintains cell stemness through the interactions with TGF- $\beta 1$. Biosci Rep 39: BSR20181525, 2019.

This work is licensed under a Creative Commons Attribution-NonCommercial-NoDerivatives 4.0 International (CC BY-NC-ND 4.0) License. 